\section{A Política de Recompensa e Promoção Influencia a Motivação do Trabalhador?}

\author{
Marcio Gonçalves de Pinho, Tara Keshar Nanda Baidya, Marta Correa \\ Dalbem e Eduardo Henrique de Sousa Salvino
}

\section{RESUMO}

Esse trabalho estuda a influência da política de recompensa e promoção na motivação do trabalhador, tanto intrínseca, entendida como própria do indivíduo, como extrínseca, vista na literatura como dependente das intervenções ou incentivos externos vinculados ao trabalho. Sendo assim, nesta pesquisa busca-se analisar o nível de motivação do trabalhador sob esses dois conceitos, que são as variáveis dependentes no trabalho. A revisão de literatura aborda teorias com importantes discussões sobre motivação no trabalho, bem como as características utilizadas nas regras de uma política de recompensa e promoção do trabalhador, sendo elas Transparência, Justiça e Controlabilidade, as quais são as variáveis independentes no trabalho. Um questionário estruturado permitiu coletar os dados e examinálos por meio da análise fatorial e regressão linear múltipla. Os resultados mostraram que a justiça empregada nas regras da recompensa monetária é uma característica que exerce influência na motivação intrínseca e extrínseca, estatisticamente significativa e relevante, já a controlabilidade do sistema de promoção exerce influência na motivação extrínseca. Contudo, algumas variáveis não alcançaram significância estatística ao ponto de causar influência na motivação. O trabalho permitiu confirmar discussões teóricas, além de oferecer um modelo de análise com visões práticas e gerenciais do cotidiano organizacional.

Palavras-chave: Motivação Extrínseca; Motivação Intrínseca; Satisfação no Trabalho; Política de Recompensa; Economia do Trabalho.

\section{Do the reward and the promotion policies influence the worker's motivation?}

\section{ABSTRACT}

This paper studies the influence of reward and promotion policy on worker motivation, both intrinsic, understood as belonging to the individual, and extrinsic, seen in the literature as dependent on interventions or external incentives linked to work. In this research, we seek to analyze the level of motivation of the worker under these two concepts, which are the dependent variables. The literature review adresses theories with important discussions about motivation at work, as well as the characteristics used in the rules of a worker's reward and promotion policy, being Transparency, Justice and Controllability, which are the independent variables. A structured questionnaire was used to collect the data. They were examined through factorial analysis and multiple linear regression. The results showed that the justice used in the rules of monetary reward is a characteristic that exerts influence in both the intrinsic and extrinsic motivation. The controllability of the promotion system exerts influence on the extrinsic motivation. However, other variables did not reach statistical significance. This work allowed us to confirm theoretical discussions. Also, it offered a model of analysis with practical and managerial visions of the organizational daily life.

Keywords: Extrinsic Motivation; Intrinsic Motivation; Job Satisfaction; Reward Policy; Labor Economics.

Recebido em: 28/07/2017 Aprovado em: 28/05/2018 Última Modificação: 21/06/2018

Check for updates

Marcio Gonçalves de Pinho (iD,

Universidade Estácio de Sá, Brasil. Mestre em Administração, UniGranRio, Brasil.

marcio.pinho1211@gmail.com

Tara Keshar Nanda Baidya (iD,

Professor Adjunto do Programa de Pós-Graduação em Administração da UniGranRio, Brasil.

Doutorado em Administração de Empresas, University of California Berkeley, Estados Unidos da América

tarabaidya@yahoo.com.br

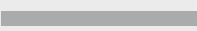

Marta Correa Dalbem (iD, Consultora Independente. Doutora em Administração, PUC-Rio, Brasil.

martadalbem@hotmail.com

Eduardo Henrique de Sousa Salvino (iD), PUC-Rio, Brasil. Mestre em Engenharia de Produção, PUC-Rio, Brasil.

esalvino@puc-rio.br 
Introdução

Estudos sobre motivação têm se mostrado de grande interesse, haja vista a quantidade de discussões na literatura sobre o tema, dentre elas a afirmação de Jenkins, Mitra, Gupta e Shaw (1998) sobre a carência de estudos sistemáticos da relação entre incentivos financeiros e a forma como o trabalhador se comporta, como também a de Cerasoli, Nicklin e Ford (2014), os quais perceberam a inexistência de abordagens empíricas de cunho prático e teórico, definindo o real papel dos incentivos extrínsecos versus motivação intrínseca. Há lacunas a serem exploradas no tema motivação, tanto no ambiente de trabalho como nos estudos organizacionais (Herpen, 2007; Kanfer, Chen, \& Pritchard, 2008), o que justifica a contínua busca por respostas para questões complexas sobre a natureza humana (Broxado, 2001).

Nesse cenário, incentivos financeiros e não financeiros se somam ao custo do trabalho, na busca de se alcançar resultados e competências (Assis, 2011), demandando da empresa repartir uma parcela do lucro como forma de estimular o trabalhador a alcançar maiores níveis de produção (Lacombe, 2005; Marras, 2009). Tal ação pode ser administrada por um sistema de recompensa e promoção, o que é visto por algumas organizações como nova vantagem competitiva (Wood \& Picarelli, 2004), pois as relações de trabalho são essenciais para o cumprimento eficaz dos objetivos organizacionais, sabendo-se que o equilíbrio entre recompensas e orçamento empresarial é uma restrição (Ulrich, 2000).

Grande parte das organizações têm sido questionadas sobre a forma como remuneram seus trabalhadores, sendo que muitas já perceberam a necessidade de readequação das suas regras frente às expectativas dos trabalhadores (Copelli \& Piccinini, 2003). Tal fato tem impulsionado as organizações a experimentarem novos modos de alcançar resultados, pondo em prática ações que proporcionem bem-estar e satisfação ao trabalhador, além de um agradável e justo ambiente de trabalho (Beuren, Santos, Marques, \& Resendes, 2017).

Verifica-se que a literatura sobre motivação é robusta, apresentando inclusive pesquisas empíricas. No entanto, há poucas inferências de cunho quantitativo e com resultados que se aproximem mais do cotidiano organizacional. Tal observação serviu de inspiração para a construção dessa pesquisa, a qual assumiu como objetivo responder a seguinte questão: A política de recompensa e promoção influencia a motivação do trabalhador? Para tanto, aqui se buscou analisar a influência de determinada política de incentivos na motivação, por meio de análises quantitativas e pautadas na percepção do trabalhador, considerando as características apontadas na literatura como sendo requisitos indispensáveis de um sistema de recompensa e promoção do trabalhador, que são "transparência", "justiça" e "controlabilidade". 


\section{Quadro Teórico-Empírico}

\section{Motivação}

Cada vez mais as organizações são desafiadas pela competitividade imposta pelo mercado e por isso buscam motivar seus trabalhadores ao alcance da produtividade desejada. Entretanto, pessoas têm suas próprias vontades e objetivos e nem sempre se sentem sensíveis ou motivadas pelos incentivos ofertados por suas empresas, o que contribui para mudanças contínuas das regras no trato com as pessoas no ambiente de trabalho.

Tal conflito nos remete aos conceitos de motivação no ambiente de trabalho, que são: a Motivação Extrínseca (ME), representada pelos incentivos ou estímulos externos que buscam influenciar o indivíduo a um determinado comportamento (Mitchell, Holton, Lee, Sablynski, \& Erez, 2001) e a Motivação Intrínseca (MI), proveniente do indivíduo, fazendo com que o mesmo se comporte de acordo com sua própria energia, sendo, portanto, liberada de dentro para fora (Broxado, 2001).

Na busca por compreender tal complexidade, o tema motivação tem sido alvo de diversos estudos ao longo do tempo, dentre os quais está nossa pesquisa, que resgata as discussões propostas por Herzberg (1968), Bandura (1989), Jenkins et al. (1998), Ryan e Deci (2000), Gagné e Deci (2005) e Frey (2012).

Herzberg (1968), ao formular a Teoria dos Dois Fatores (higienemotivação), postulou que fatores geradores da insatisfação não são os mesmos geradores da satisfação no trabalho. Fatores como política da organização, relacionamento com o supervisor e salário, denominados "fatores de higiene", não tornam um trabalhador satisfeito, podendo ser apenas capazes de não o desmotivar, ao passo que a realização de uma tarefa desafiadora, responsabilidade, reconhecimento e crescimento são fatores que causam satisfação.

Bandura (1989), complementando o estudo, propôs a Teoria da Autoeficácia, definindo o ser humano como um agente capaz de exercer controle sobre suas emoções, pensamentos, ações e seu ambiente. Desta forma, a autoeficácia conduz o indivíduo a executar uma tarefa desafiadora desde que com alto grau de certeza sobre sua capacidade para tal, ou seja, o trabalhador tem seu nível de motivação determinado pela forma como ele encara o desafio do trabalho.

Nessa perspectiva, motivação é vista como uma escolha por comportamentos voltados para objetivos, sendo relacionada a dois fatores: 1) expectativas de resultados, sob a ótica de que determinados comportamentos se tornam úteis para alcançar certos objetivos e, 2) crenças de autoeficácia, referindo-se às percepções que cada um tem para promover ações voltadas ao alcance dos objetivos. O emprego de ações que levem ao alcance de objetivos desafiadores faz com que determinadas pessoas tenham desempenho melhor do que outras com objetivos menos desafiadores (Fontaine, 2005). 
Alcançar melhor desempenho depende, então, da energia que o trabalhador está disposto a empregar na sua tarefa, da sua vontade, persistência e intenção. Por essa razão, constantemente a motivação se encontra no centro das discussões no campo da psicologia do trabalho, sendo valorizada pelas consequências que pode causar, pois "a motivação produz" (Ryan \& Deci, 2000).

Ryan e Deci (2000) acreditam que as pessoas podem se sentir envolvidas e proativas em suas tarefas no trabalho, como também podem, alternativamente, serem passivas ealienadas. Esses autores, ao discutira Teoria da Autodeterminação, projetaram a ideia de que determinadas condições podem elevar ou diminuir a motivação intrínseca (ou automotivação). Eles pontuam que fatores como autonomia e competência, quando satisfeitos, são capazes de elevar a motivação intrínseca e o bem-estar, mas quando frustrados, o efeito se inverte.

Frey (2012), enriquecendo esse campo de estudo, denominou de "Crowding Effect" a mudança do locus da percepção de causalidade, indicando que: (i) estando sob controle externo e privado de demonstrar autodeterminação para a tarefa, o trabalhador reagirá reduzindo sua motivação intrínseca e, portanto, seu esforço; (ii) se a intervenção externa for percebida de forma positiva e como suporte, então promoverá sua autodeterminação para a tarefa e, consequentemente, sua motivação intrínseca. Desta forma, a motivação intrínseca pode ser afetada de forma negativa, chamada de efeito "crowding-out", ou de forma positiva, o efeito "crowding-in". Nesse sentido, fatores externos como incentivos tangíveis, vigilância e prazos tendem a afetar o sentimento de autonomia, mudando o locus da energia para a tarefa de interno para externo, reduzindo a motivação intrínseca. Por outro lado, quanto maior a autonomia, maior a motivação intrínseca (Ryan \& Deci, 2000, Gagné \& Deci, 2005; Frey, 2012).

\section{Recompensa e Promoção}

Recompensa pode ser determinada mensalmente por um valor fixo ou variável, incluindo-se os benefícios. O salário é visto pelo trabalhador como a parte fixa da recompensa e sua principal ou única fonte de sobrevivência familiar e, portanto, determinante da sua condição de vida. Já a parte variável pode ser composta por valores como comissões, incentivo por desempenho ou participação nos resultados (Vizioli, 2010; Assis, 2011).

Para recompensar e promover por desempenho, Bergamini (2012) ressalta a importância de se utilizar uma adequada avaliação por meio de um instrumento confiável e objetivo, sensível ao ponto de detectar diferenças pessoais, de modo a neutralizar tendências pessoais do avaliador. Em resumo, o instrumento deve ser válido tanto em termos técnicos quanto éticos.

Já os benefícios surgiram nas pautas de reivindicações sindicais, sob a justificativa de que os trabalhadores se dedicam diariamente aos objetivos organizacionais, sendo, portanto, merecedores de incentivos além da recompensa, algo que contribua para a manutenção da sua saúde e segurança, o que, em certa medida, também proporciona vantagens para a empresa que passa a contar com pessoas motivadas e focadas no trabalho (Vizioli, 2010, p. 184). 
Na visão de Bonilla, Vaz e Selig (2012), as recompensas encontram embasamento nas teorias motivacionais desenvolvidas por estudiosos da psicologia do trabalho, tendo como principal propósito motivar o empregado a trabalhar movido por estímulos de natureza intrínseca ou extrínseca. Para Lacombe (2005), um sistema de recompensa e promoção deve ter por objetivo estabelecer adequados incentivos aos que estão comprometidos com a organização, devendo apresentar coerência interna e externa. Em outras palavras, a política de incentivos deve ser percebida como justa, capaz de gerar equidade salarial para o trabalhador em relação aos seus pares internos e ao mercado.

Através da equidade interna, a empresa deve assegurar uma forma de correlacionar os cargos por sua complexidade e promover uma avaliação, classificação e pontuação de cada cargo, considerando fatores tais como experiência, escolaridade e complexidade das funções (Assis, 2011). Assim, será possível determinar seu peso ou valor relativo na engrenagem organizacional (Pontes, 2007).

Com a equidade externa a empresa pode estabelecer uma política salarial adequada ao que se observa no mercado, em cargos similares. A falta da equidade externa também pode gerar insatisfação entre os trabalhadores, culminando com a perda de bons profissionais para organizações com melhor política salarial (Pontes, 2007, p. 35).

Para Jenkins et al. (1998), as organizações deveriam dar maior atenção sobre a forma como estão conduzindo sua política de incentivos financeiros, pondo em prática certas reflexões, tais como: estamos desperdiçando dinheiro? Devemos revisar nossa política de incentivos?

Paschoal (2007, pp. 92-93) assegura que são necessárias algumas características para que o sistema de recompensa e promoção seja eficaz, como: objetividade, clareza, alinhamento com o negócio e integração com as demais políticas e filosofias da organização, além de flexibilidade (porém controle), condições de atrair, reter e motivar. Quando a empresa alcança esse estágio, tende a cultivar a credibilidade e a oportunidade de crescimento.

Segundo Pontes (2007), a oportunidade de crescimento profissional é uma das formas de se reconhecer e recompensar o desempenho do trabalhador. Além disso, controlabilidade se refere à percepção do trabalhador de que ao aplicar maior esforço alcançará maior recompensa, o que o mantém com certo grau de autonomia (Herpen, 2007).

Assis, Araujo, Fernandes e Freitas (2017) mostram diversas visões sobre os termos recompensar e reconhecer, dentre elas a ideia de que recompensas devem estar alinhadas com as metas organizacionais, na medida em que podem tanto funcionar como reforço auxiliar do comportamento desejado, quanto ser um desestímulo de comportamentos inadequados. Já o reconhecimento tende a reforçar valores organizacionais, inclusive promovendo ações de homenagem que destacam o bom trabalhador entre seus pares, sem necessariamente haver incentivo financeiro para tal. Para esses autores, a organização deve se valer de uma gestão de pessoas capaz de mobilizar a força de trabalho com uso de mecanismos de recompensa e reconhecimento, de incentivos financeiros e não financeiros. 
À luz dessas características apontadas pela literatura como desejáveis em uma política dessa natureza, Herpen (2007) pesquisou os efeitos da política de recompensa e promoção de uma organização holandesa na motivação de seus trabalhadores. O autor concluiu que oportunidades de promoção afetam positivamente a motivação intrínseca e que a justiça do sistema tem uma relação significativa com a motivação extrínseca.

De acordo com Herpen (2007), estudos da Psicologia e da Economia ainda não encontraram uma resposta unívoca para os efeitos da recompensa monetária e promoção na motivação dos trabalhadores, tendo grande parte da literatura econômica se voltado para o desenvolvimento de modelos teóricos, sem investigar empiricamente suas implicações.

Tais observações serviram de inspiração para esta pesquisa, a qual buscou investigar empiricamente o tema. Utilizou-se como exemplo o trabalho de Herpen e para se alcançar os objetivos propostos foram formuladas as seguintes hipóteses:

H1a: A transparência da política de compensação monetária influencia a motivação extrínseca.

H1b: A justiça da política de compensação monetária influencia a motivação extrínseca.

H1c: A controlabilidade da política de compensação monetária influencia a motivação extrínseca.

H2a: A transparência da política de promoção influencia a motivação extrínseca.

H2b: A justiça da política de promoção influencia a motivação extrínseca. H2c: A controlabilidade da política de promoção influencia a motivação extrínseca.

H3a: A transparência da política de compensação monetária influencia a motivação intrínseca.

H3b: A justiça da política de compensação monetária influencia a motivação intrínseca.

H3c: A controlabilidade da política de compensação monetária influencia a motivação intrínseca.

H4a: A transparência da política de promoção influencia a motivação intrínseca.

H4b: A justiça da política de promoção influencia a motivação intrínseca. H4c: A controlabilidade da política de promoção influencia a motivação intrínseca.

Como é possível observar, as hipóteses acima se destinam a refutar (ou a falhar em refutar) que uma determinada política de recompensa e promoção exerce influência na motivação extrínseca e na motivação intrínseca do trabalhador.

\section{Procedimentos Metodológicos}

\section{Dados e amostra}

O local de pesquisa escolhido é uma indústria farmacêutica de porte médio, fundada no Brasil na primeira metade do século XX, localizada na cidade do Rio de Janeiro. A escolha da empresa seguiu o critério de acessibilidade 
pela facilidade de se aproximar dos que decidem e implementam a política de recompensa e promoção, como também dos trabalhadores afetados por tal política.

A amostra alcançou 160 trabalhadores, sendo 145 ocupantes de cargos operacionais e 15 de gestão, representando 63,49\% dos trabalhadores da empresa. Tal amostra atende ao recomendado para se empreender uma análise fatorial, isto é, representa mais do que 5 vezes o número de variáveis do modelo (Hair, Black, Babin, Anderson \& Tatham, 2009).

\section{Variáveis}

\section{Variável dependente: Motivação}

Este trabalho utilizou um questionário estruturado contendo 24 variáveis em escala Likert de 5 pontos (1-discordo, 2-discordo em parte, 3-não concordo nem discordo, 4-concordo em parte e 5-concordo) para levantar as percepções e medir níveis motivacionais dos trabalhadores.

As variáveis utilizadas na presente pesquisa baseiam-se em grande parte no trabalho de Herpen (2007), as quais se destinam a levantar as percepções quanto ao nível de motivação extrínseca, sendo elas: (V11) Minha compensação me deixa motivado(a) a fazer o máximo esforço possível; (V12) Há chances suficientes de promoção, o que me estimula a trabalhar muito; (V13) Estou satisfeito(a) com a forma de como minha compensação é definida; (V14) Estou satisfeito(a) com as chances de promoção que existem na empresa; (V15) A empresa considera importante ter um sistema de compensação bem definido e claro para todos; (V16) A empresa considera importante ter um sistema de promoção bem definido e claro para todos; (V17) Estou entusiasmado(a) com o meu salário; (V18) Acho que o sistema de compensação e promoção me motiva.

As variáveis destinadas a levantar as percepções quanto ao nível de motivação intrínseca são: (V19) Estou muito satisfeito(a) com o trabalho que faço; (V20) Meu trabalho vale a pena o esforço; (V21) Estou satisfeito(a) com meu emprego; (V22) Muitas vezes tenho que me forçar a ir trabalhar; (V23) Normalmente estou entusiasmado(a) com meu emprego e (V24) No trabalho muitas vezes me sinto como se o dia nunca fosse acabar.

Os coeficientes de correlação superaram 0,30 em sua maioria e o teste de $\mathrm{KMO}$ alcançou 0,85 , indicando que tanto o padrão de correlação é aceitável (Figueiredo \& Silva, 2010) quanto o teste de KMO, confirmando que a base de dados é adequada ao uso da Análise Fatorial (Malhotra, 2012).

Quanto ao número de fatores, verificou-se que somente dois apresentam autovalor acima de 1, sendo o Fator 1, 6,180828 (44,15\%) e o Fator 2, 2,326516 (16,62\%), correspondendo ambos por 60,77\% da variância, de modo que a análise prossegue considerando apenas esses dois fatores.

A Tabela 1 apresenta as relações após a rotação Varimax dos fatores e identifica quais variáveis estão mais claramente ligadas a cada um dos dois fatores. 
Tabela 1: Matriz Fatorial Rotacionada (Varimax), construto Motivação

\begin{tabular}{lcc}
\hline \multicolumn{1}{c}{ Variáveis } & \multicolumn{2}{c}{ Motivação } \\
\hline \multicolumn{1}{c}{ Extrinseca } & Intrinseca \\
\hline V11: Minha compensação me deixa motivado(a) a fazer o máximo esforço possível. & $\mathbf{0 , 6 9}$ & $-0,34$ \\
V12: Há chances suficientes de promoção, o que me estimula a trabalhar muito. & $\mathbf{0 , 7 2}$ & $-0,21$ \\
V13: Estou satisfeito(a) com a forma de como minha compensação é definida. & $\mathbf{0 , 6 4}$ & $-0,32$ \\
V14: Estou satisfeito(a) com as chances de promoção que existem na empresa. & $\mathbf{0 , 7 7}$ & $-0,2$ \\
V15: A empresa considera importante ter um sistema de compensação bem definido e claro & $\mathbf{0 , 8 4}$ & 0,07 \\
para todos. & & \\
V16: A empresa considera importante ter um sistema de promoção bem definido e claro & $\mathbf{0 , 8 2}$ & 0,03 \\
para todos. & $\mathbf{0 , 5 7}$ & $-0,38$ \\
V17: Estou entusiasmado(a) com o meu salário. & $\mathbf{0 , 8}$ & $-0,23$ \\
V18: Acho que o sistema de compensação e promoção me motiva. & 0,22 & $\mathbf{- 0 , 8}$ \\
V19: Estou muito satisfeito(a) com o trabalho que faço. & 0,29 & $\mathbf{- 0 , 8 2}$ \\
V20: Meu trabalho vale a pena o esforço. & 0,32 & $\mathbf{- 0 , 7 4}$ \\
V21: Estou satisfeito(a) com meu emprego. & $-0,03$ & $\mathbf{0 , 6 8}$ \\
V22: Muitas vezes tenho que me forçar a ir trabalhar. & 0,16 & $\mathbf{- 0 , 8 1}$ \\
V23: Normalmente estou entusiasmado(a) com meu emprego. & $-0,04$ & $\mathbf{0 , 6 4}$ \\
V24: No trabalho muitas vezes me sinto como se o dia nunca fosse acabar. & 0,142441 & 0,018329 \\
Escore Fatorial: & 0,074304 & $a-0,20093$ \\
Alpha de Cronbach & 0,90 & 0,86 \\
\hline
\end{tabular}

Fonte: Dados da pesquisa.

Com a obtenção dos escores fatoriais, foram calculados os valores de ME (Fator 1) e MI (Fator 2) a partir das respostas dadas por cada respondente às variáveis V11 a V24. Portanto, ME e Ml foram contruídos da seguinte forma:

$$
\begin{aligned}
& \mathrm{ME}_{i}=\alpha_{11} \mathrm{~V}_{11 i}+\ldots+\alpha_{24 i} \mathrm{~V}_{24 i}=0.142441 \mathrm{~V}_{11 i}+\ldots+0,074304 \mathrm{~V}_{24 i} \\
& \mathrm{Ml}_{i}=\theta_{11} \mathrm{~V}_{11 i}+\ldots+\theta_{24} \mathrm{~V}_{24 i}=0,018329 \mathrm{~V}_{11 i}+\ldots-0,20093 \mathrm{~V}_{24 i} \\
& \text { em que: }
\end{aligned}
$$

$\mathrm{ME}_{i}$ e $\mathrm{Ml}_{i}$ referem-se à motivação extrínseca e intrínseca, respectivamente, do respondente i (i $[1,2, \ldots ., 160])$, conforme calculado pelo modelo fatorial adotado; $\alpha_{11}, \ldots, \alpha_{24 i}$ referem-se aos escores fatoriais obtidos para ME (Fator 1) e $\theta_{11}, \ldots, \theta_{24}$ referem-se aos escores fatoriais obtidos para Ml (Fator 2). $V_{i j}$ é a resposta dada à variável $j,(j \in[11,12, \ldots, 24])$ pelo respondente $\mathrm{i}(\mathrm{i} \in$ $[1,2, \ldots, 160])$.

Os valores gerados para os construtos Motivação Extrínseca (ME) e Motivação Intrínseca (MI) ficaram nos intervalos [0,80; 6,74] e [(2,24); $(3,95)]$, respectivamente. Importante lembrar que ME e Ml são as variáveis dependentes na regressão contra os construtos Transparência, Justiça e Controlabilidade, os quais também foram previamente analisados por AF. 
Variáveis Independentes

As variáveis destinadas a levantar as percepções quanto à Transparência das regras são: (V1) Para mim é muito clara a forma como minha compensação é definida; (V2) Sei claramente quais são minhas chances de promoção e (V3) Para mim são claros os critérios de promoção de cargo.

Quanto à Justiça das regras as variáveis são: (V4) Minha compensação faz com que me sinta valorizado(a) pelo meu trabalho; (V5) Minha compensação é compatível com meu desempenho; (V6) Meu salário é bom, quando comparado com o que eu poderia ganhar em outra empresa, fazendo o mesmo trabalho; (V7) Eu acho justa a política de compensação da empresa e (V8) Pessoas que foram promovidas na empresa, fizeram por merecer.

Quanto à Controlabilidade das regras as variáveis são: (V9) Posso aumentar a minha compensação total, trabalhando muito e (V10) Minhas chances de ser promovido(a) dependem mais de mim.

A AF permitiu ainda obter dois outros construtos que ajudaram a descrever a política de recompensa, que são: TP (Transparência na Promoção), formado a partir da combinação das variáveis V2 e V3, e JCM (Justiça na Compensação Monetária), formado a partir das variáveis V4 a V7.

O processo foi semelhante, isto é, após a construção da matriz de correlação e rotação dos fatores, foram obtidos os escores fatoriais (Tabelas 2 e 3) desses dois fatores explicativos da motivação.

Tabela 2. Matriz dos Escores Fatoriais (V2 e V3), construto TP

\begin{tabular}{cc}
\hline Variável & Fator $\mathbf{1}$ \\
\hline V2 & 0,5707 \\
V3 & 0,5707 \\
\hline
\end{tabular}

Nota. Dados da pesquisa.

Tabela 3. Matriz dos Escores Fatoriais (V4 a V7), construto JCM

\begin{tabular}{cc}
\hline Variável & Fator 1 \\
\hline V4 & 0,340607 \\
V5 & 0,330168 \\
V6 & 0,297205 \\
V7 & 0,280459 \\
\hline
\end{tabular}

Nota. Dados da pesquisa.

Os valores de TP e JCM a serem transportados para a fase de regressão são, portanto, os seguintes:

$$
\begin{aligned}
& \mathrm{TP}_{i}=\delta_{2} \mathrm{~V}_{2 i}+\delta_{3} \mathrm{~V}_{3 i}=0,5707 \mathrm{~V}_{2 i}+0,5707 \mathrm{~V}_{3 i} \\
& \mathrm{JCM}_{i}={ }^{\prime} \Upsilon_{4} \mathrm{~V}_{4 i}+\Upsilon_{5} \mathrm{~V}_{5 i}+{ }^{\prime} \Upsilon_{6} \mathrm{~V}_{6 i}+\Upsilon_{7} \mathrm{~V}_{7 i}=0,340607 \mathrm{~V}_{4 i}+\ldots+0,280459 \mathrm{~V}_{7 i} \\
& \text { em que: } \\
& \delta_{2}, \delta_{3} \text { são os escores fatoriais obtidos na Tabela 2; } \\
& { }^{\prime} \Upsilon_{4}, \ldots, ' \Upsilon_{7} \text { são os escores fatoriais obtidos na Tabela 3; } \\
& \mathrm{V}_{\mathrm{ji}} \text { é a resposta dada à variável j pelo respondente } \mathrm{i}(\mathrm{i} \in[1,2, \ldots, 160]) ;
\end{aligned}
$$


Estes dois construtos também apresentaram consistência interna verificada pelo alfa de Cronbach, sendo encontrado 0,70 para V2 e V3 e 0,81 para V4, V5, V6 e V7.

Finalmente, as relações de causalidade entre as variáveis independentes (V1, TP, JCM, V8, V9 e V10) e as dependentes (ME e MI) foram analisadas por meio da técnica de regressão linear múltipla, cujos resultados estão na seção 4.

\section{Método}

Foram feitas duas regressões, uma tendo ME como variável dependente, outra tendo Ml. Em cada regressão, as variáveis independentes são: Transparência da Promoção (TP) e Justiça na Compensação Monetária (JCM), obtidas por meio de análise fatorial, além das variáveis observadas Transparência da Recompensa Monetária (V1), Justiça da Promoção (V8), Controlabilidade da Recompensa Monetária (V9) e Controlabilidade da Promoção (V10).

Em resumo, para testar as hipóteses de que cada variável independente influencia $\mathrm{ME}$ e/ou MI, foram utilizados os seguintes modelos de regressão:

$$
\begin{aligned}
& \mathrm{ME}=\beta_{0}+\beta_{1} \mathrm{X}_{1 i}+\beta_{2} \mathrm{X}_{2 i}+\beta_{3} \mathrm{X}_{3 i}+\beta_{4} \mathrm{X}_{4 i}+\beta_{5} \mathrm{X}_{5 i}+\beta_{6} \mathrm{X}_{6 i}+\varepsilon_{i} \\
& \mathrm{Ml}=\alpha_{0}+\alpha_{1} \mathrm{X}_{1 i}+\alpha_{2} \mathrm{X}_{2 i}+\alpha_{3} \mathrm{X}_{3 i}+\alpha_{4} \mathrm{X}_{4 i}+\alpha_{5} \mathrm{X}_{5 i}+\alpha_{6} \mathrm{X}_{6 i}+\xi_{i} \\
& \text { em que: }
\end{aligned}
$$

ME - valor atribuído à motivação extrínseca, calculado com base nas variáveis observadas V11 a V24, ponderadas pelos seus escores fatoriais;

$\mathrm{Ml}$ - valor atribuído à motivação intrínseca, calculado com base nas variáveis observadas V11 a V24, ponderadas pelos seus escores fatoriais;

$\beta_{0}, \alpha_{0}$ - interceptos;

$\beta_{i}, \alpha_{i}$ - são os coeficientes associados às variáveis Vi e aos construtos;

$\mathrm{X}_{i i}$-é a resposta dada a variável $\mathrm{j},(\mathrm{j} \in[\mathrm{V} 1, \mathrm{~V} 8, \mathrm{~V} 9$ e V10]) pelo respondente $\mathrm{i}$, bem como os valores obtidos para os fatores TPi e JCMi, (i $\in[1,2, \ldots, 160])$;

$\varepsilon_{i}, \xi_{i}$ - são os erros(resíduos).

\section{Resultados}

A Tabela 4 consolida os resultados obtidos com a regressão dos dados. Os resultados identificam que há relação estatisticamente significativa entre Motivação Extrínseca e duas características, sendo elas a Justiça na Política de Compensação Monetária $(\beta=0,49, p$-value $<0,00)$ e a Controlabilidade das oportunidades ofertadas pela Política de Promoção $(\beta=0,34, p$-value $<0,00)$, apoiando, respectivamente, as hipóteses H1b e H2c. 
Tabela 4. Regressão

\begin{tabular}{|c|c|c|c|c|c|c|c|c|}
\hline \multirow[b]{2}{*}{ Construtos } & \multicolumn{4}{|c|}{ Motivação Extrínseca } & \multicolumn{4}{|c|}{ Motivação Intrínseca } \\
\hline & Coeficiente & $\begin{array}{c}\text { Erro } \\
\text { Padrão }\end{array}$ & Teste $t$ & Valor $\mathbf{P}$ & Coeficiente & $\begin{array}{c}\text { Erro } \\
\text { Padrão }\end{array}$ & Teste $t$ & Valor $\mathbf{P}$ \\
\hline \multicolumn{9}{|l|}{ PCM } \\
\hline Transparência & 0 & 0,07 & 0 & 1 & $-0,16$ & 0,08 & $-1,9$ & 0,06 \\
\hline Justiça & 0,49 & 0,07 & 7,37 & $0,00^{\star *}$ & 0,32 & 0,08 & 4,11 & $0,00^{\star *}$ \\
\hline Controlabilidade & 0,06 & 0,06 & 1,12 & 0,26 & 0,05 & 0,07 & 0,8 & 0,42 \\
\hline \multicolumn{9}{|l|}{ PP } \\
\hline Transparência & 0,08 & 0,07 & 1,21 & 0,23 & 0,07 & 0,08 & 0,86 & 0,39 \\
\hline Justiça & 0,06 & 0,07 & 0,85 & 0,4 & 0,03 & 0,08 & 0,34 & 0,73 \\
\hline Controlabilidade & 0,34 & 0,07 & 5,16 & $0,00^{\star *}$ & $-0,1$ & 0,08 & $-1,3$ & 0,21 \\
\hline Intercepto & 0,28 & 0,53 & 0,53 & 0,6 & 0,42 & 0,63 & 0,68 & 0,5 \\
\hline $\mathrm{R}^{2}$ & 0,6 & & & & 0,33 & & & \\
\hline $\mathrm{R}^{2}$ ajustado & 0,56 & & & & 0,25 & & & \\
\hline $\mathrm{F}$ & 12,7 & & & & 4,12 & & & \\
\hline Observações & 160 & & & & 160 & & & \\
\hline
\end{tabular}

Nota. PCM (Política de Compensação Monetária). PP (Política de Promoção).

$a * * p<0,01$, bicaudal

Fonte: Dados da pesquisa.

Segundo os resultados, a Transparência, tanto da política de compensação monetária quanto da política de promoção, a Controlabilidade da política de compensação monetária e a Justiça da política de promoção, não têm efeito estatisticamente significativo na Motivação Extrínseca. Com base nesses resultados, estão refutadas as hipóteses $\mathrm{H} 1 \mathrm{a}, \mathrm{H} 1 \mathrm{c}, \mathrm{H} 2 \mathrm{a}$ e H2b.

Os resultados mostram haver relação estatisticamente significativa entre Motivação Intrínseca e a Justiça na Política de Compensação Monetária $(\alpha=0,32, p$-value $<0,00$ ), o que apoia a hipótese H3b (a justiça da política de compensação monetária influencia a motivação intrínseca).

Não alcançaram relação com a Motivação Intrínseca as características Transparência e Controlabilidade da Política de Compensação Monetária, bem como as características Transparência, Justiça e Controlabilidade da Política de Promoção. Vale lembrar que esse último quesito - Controlabilidade da Política de Promoção - foi significativo e relevante para o outro tipo de motivação, a Motivação Extrínseca. Desta forma, estão refutadas as hipóteses $\mathrm{H} 3 \mathrm{a}, \mathrm{H} 3 \mathrm{c}, \mathrm{H} 4 \mathrm{a}, \mathrm{H} 4 \mathrm{~b}$ e H4c.

\section{Conclusões}

O presente trabalho assumiu o desafio de pesquisar, analisar e responder se um determinado sistema de recompensa e promoção influencia o trabalhador de uma indústria farmacêutica brasileira, considerando os conceitos da motivação extrínseca e da motivação intrínseca no ambiente de trabalho. 
A análise permitiu identificar que três atributos do modelo exercem influência na motivação. Destes atributos que tiveram significância estatística, a justiça percebida do sistema de recompensa foi o único a ter impacto tanto na motivação extrínseca quanto na motivação intrínseca. Já a controlabilidade do sistema de promoção exerce influência apenas na motivação extrínseca. Ambas influências são relevantes, em especial para a motivação extrínseca, confirmando o estudo de Herpen (2007) em empresa holandesa.

Há que se levar em conta que, na empresa brasileira pesquisada, a compensação monetária também ocorre pela oferta de benefícios discricionários e pagamento de participação nos lucros, atraentes aos olhos dos seus trabalhadores ao ponto de retê-los, dado que a rotatividade é baixa, como já previa Vieira e Silva (2008) ao analisar práticas de blindagem organizacional.

À luz da teoria de Herzberg (1968), verifica-se que os resultados da pesente análise indicam que a transparência do sistema de compensação monetária e promoção, justiça na promoção e controlabilidade do sistema de compensação monetária se traduzem em fatores capazes apenas de não causar desmotivação nos trabalhadores pesquisados. Verifica-se, ainda, que os mesmos trabalhadores percebem como justa a compensação monetária, bem como reportaram controlabilidade do sistema de promoção e que, mais importante, esses dois quesitos influenciam na sua motivação.

Os mesmos resultados confirmam a proposta de Bandura (1989), ou seja, o trabalhador é capaz de controlar seus pensamentos, emoções e ações, permanecendo indiferente diante de certos incentivos ofertados pela organização, podendo, por outro lado, se sentir motivado a agir quando percebe justiça entre o que faz e o que recebe em troca, praticando sua autoeficácia em comportamentos orientados para objetivos. Nessa mesma direção, também se verifica a confirmação de que a política de recompensa da empresa pesquisada está deixando a desejar ao frustrar a autodeterminação dos seus trabalhadores em certos aspectos, fazendo com que as atuais regras tenham pouco efeito na motivação intrínseca (Ryan \& Deci, 2000).

Outra confirmação revelada nos resultados é o fato de que, quanto maior a transparência da compensação monetária, menor tende a ser a motivação intrínseca, embora o impacto seja pequeno $(\alpha=-0,16$; $p$-value $=0,06)$. Esse resultado é de certa forma surpreendente, porém corrobora as ideias fundamentadas por Frey (2012), ao descrever o efeito crowding - a mudança do locus da percepção de causalidade, ou seja, o trabalhador tem sua motivação intrínseca afetada ao sentir que seu empenho espontâneo em determinada tarefa não está sendo apreciado.

Como contribuições acadêmicas, mas sem pretender esgotar o tema, este trabalho corrobora fundamentações teóricas, bem como estudos sobre motivação no trabalho, especialmente o de Herpen (2007), Gagné e Déci (2005) e Frey (2012). Além disso, o modelo de análise aplicado nesse trabalho oferece um ferramental com visões mais próximas do cotidiano organizacional, útil na criação de indicadores para o processo decisório no campo da gestão de pessoas, uma vez que grande parte da literatura tem se voltado para estudos que, mesmo empíricos, não fazem a análise quantitativa dos dados. 
Como principal limitação, a pesquisa encontrou dificuldade de acesso aos trabalhadores atuantes fora da sede da empresa, em especial os do setor de vendas, o que dificultou ampliar a amostra. Mesmo assim, a amostra alcançada foi suficiente para permitir análises estatísticas.

Finalizando, este trabalho propõe três sugestões para novas pesquisas: (i) repetir a pesquisa na mesma indústria, buscando ampliar a amostra e incluir outros extratos da empresa; (ii) buscar outra empresa que tenha em vigor uma política de cargos e salários considerada estratégica e replicar o estudo e; (iii) replicar o estudo em empresas de setores, tamanhos e culturas diferentes, buscando conclusões generalizáveis.

\section{Agradecimentos}

Os autores desse trabalho agradecem ao editor da Revista Eletrônica de Ciência Administrativa (RECADM), bem como aos seus pereceristas, os quais, mesmo que de forma anônima, contribuíram com relevantes sugestões e recomendações ao enxergarem o esforço, resultados e importância dessa pesquisa para o tema tratado, o que proporcionou preencher algumas lacunas e finalizar esse trabalho com melhor exposição das ideias.

\section{Referências}

Assis, M. T. (2011). Gestão de programas de remuneração: conceitos, aplicações e reflexões: visão generalizada dos programas de aplicações. Rio de Janeiro: Qualitymark.

Assis, M. T., Araujo, F. F., Fernandes, V. C., \& Freitas, J. A. S. B. E. (2017, outubro). Recompensas e reconhecimento: a literatura e a visão de executivos de recursos humanos. Anais do Encontro Nacional da Associação Nacional de Pós-Graduação e Pesquisa em Administração, São Paulo, SP, Brasil, 41.

Bandura, A. (1989). Human Agency in Social Cognitive Theory. American Psychologist, 44(9), 1175-1184.

Bergamini, C. W. (2012). Avaliação de desempenho humano na empresa. (4a ed). São Paulo: Atlas.

Beuren, I. M., Santos, V. D., Marques, L., \& Resendes, M. (2017). Relação entre percepção de justiça organizacional e satisfação no trabalho. Revista de Educação e Pesquisa em Contabilidade, 11(4), 69-86.

Bonilla, M. A. M., Vaz, C. R., \& Selig, P. M. (2012, junho). Indicadores de desempenho em recompensas monetárias e não-monetárias: seleção de um referencial teórico de pesquisa e análise bibliométrica. Anais do VIII Congresso Nacional de Excelência em Gestão, Rio de Janeiro, RJ, Brasil, 8.

Broxado, S. (2001). A verdadeira motivação na empresa: entendendo a psicologia organizacional e dicas para a motivação no dia-a-dia das empresas. Rio de Janeiro: Qualitymark. 
Cerasoli, C. P., Nicklin, J. M., \& Ford, M.T. (2014). Intrinsic motivation and extrinsic incentives jointly predict performance: a 40-year meta-analysis. Psychological Bulletin, 140(4), 980-1008.

Copelli, F. L., \& Piccinini, V. C. (2003). Sistema de remuneração por habilidades para trabalhadores multifuncionais. REAd - Revista Eletrônica de Administração, 9(2), 1-18.

Figueiredo, D. B., Filho, \& Silva, J. A., Jr. (2010). Visão além do alcance: uma introdução à análise fatorial. Campinas: Opinião Pública, 16(1) 160-185.

Fontaine, A. M. (2005). Motivação em contexto escolar. Lisboa: Universidade Aberta.

Frey, B. S. (2012). Crowding out and crowding in of intrinsic preferences. In: Massachusetts Institute of Technology. (Ed.). Reflexive governance of global public goods, 4(6), 74-83.

Gagné, M., \& Deci, E. L. (2005). Self-determination theory and work motivation. Journal of Organizational Behavior, 26, 331-362.

Hair, J. F., Jr., Black, W. C., Babin, B. J., Anderson, R. E., \& Tatham, R. L. (2009). Análise multivariada de dados. (6 $6^{\mathrm{a}}$ ed). Porto Alegre: Bookman.

Herpen, M. F. M., Van. (2007). Pay, promotions, and performance: essays on personnel economics s.n. Tese de doutorado, University of Groningen, Netherlands.

Herzberg, F. (1968). One more time: how do you motivate employees? Harvard Business Review. January-February. 46, 53-62.

Jenkins, G. D., Jr., Mitra, A., Gupta, N., \& Shaw, J. D. (1998). Are financial incentives related to performance? A Meta-Analytic Review of Empirical Research. Journal of Applied Psychology, 83(5), 777-787.

Kanfer, R., Chen, G., \& Pritchard, R. D. (2008). Work motivation: Forging new perspectives and directions in the post-millennium. In Kanfer, K., G. Chen, G., \& Pritchard, R. D. (Eds.), Work motivation: Past, present, and future (pp. 601-631). New York: Taylor \& Francis.

Lacombe, F. J. M. (2005). Recursos humanos: princípios e tendências. São Paulo: Saraiva.

Malhotra, N. K. (2012). Pesquisa de marketing: uma orientação aplicada. (6ª ed). Porto Alegre: Bookman.

Marras, J. P. (2009). Administração de recursos humanos: do operacional ao estratégico. São Paulo, Saraiva.

Mitchell, T. R., Holton, B. C., Lee, T. W., Sablynski, C. J., \& Erez, M. (2001). Why people stay: using job embeddedness to predict voluntary turnover. Academy of Management Journal, 44, 1102-1122.

Paschoal, L. (2007). Administração de cargos e salários: manual prático e novas metodologias. ( $\left.3^{\mathrm{a}} \mathrm{ed}\right)$. Rio de Janeiro: Qualitymark.

Pontes, B. R. (2007). Administração de cargos e salários: carreira e remuneração. (12 ed). São Paulo: LTR.

Ryan, R. M., \& Deci, E. L. (2000). Self-Determination Theory and the Facilitation of Intrinsic Motivation, Social Development, and Well-Being. American Psychologist, 55(1), 68-78. 
Revista Eletrônica de Ciência Administrativa

409

Ulrich, D. (2000). Os campeões de recursos humanos. (4a ed). São Paulo: Futura.

Vieira, A., \& Silva, C. J. M. (2008). Por que deixar a empresa quando tudo indica que o melhor é ficar? Revista de Ciências da Administração, 10(20), 37-58.

Vizioli, M. (2010). Administração de recursos humanos. São Paulo: Pearson.

Wood, T., Jr., \& Picarelli, V., Filho. (2004). Remuneração estratégica: a nova vantagem competitiva. ( $\left.3^{\mathrm{a}} \mathrm{ed}\right)$. São Paulo: Atlas. 